\title{
ITERATIVE METHODS FOR NONLINEAR QUASI COMPLEMENTARITY PROBLEMS
}

\author{
MUHAMMAD ASLAM NOOR \\ Mathematics Department, College of Science \\ King Saud University, Riyadh 11451 , Saudi Arabia.
}

(Received April 13, 1985 and in revised form July 1, 1986)

\begin{abstract}
In this paper, we consider and study an iterative algorithm for finding the approximate solution of the nonlinear quasi complementa-rity problem of finding $u \in K(u)$, such that$$
T u \varepsilon K^{\star}(u) \text { and }(u-m(u), T u)=0
$$

where $m$ is a point-to-point mapping, $T$ is a (nonlinear) continuous mapping from a real Hilbert space $H$ into itself and $K^{*}(u)$ is the polar cone of the convex cone $\mathrm{K}(\mathrm{u})$ in $\mathrm{H}$. We also discuss the convergence criteria and several special cases, which can be obtained from our main results.
\end{abstract}

KEY WORDS AND PHRASES. Complementarity problems, Variational inequalities, Iterative methods, Converaence criteria.

1980 MATHEMATICS SUBJECT CLASSIFICATION CODES. 90C33, 49D20,65K10.

1. INTRODUCTION.

The relationship between variational inequalities and complementarity is well known. Variational inequalities have been extended and generalized in various directions to study a wide class of difficulty problems arising in different branches of mathematical and engineering sciences. Bensoussan and Lions [1] introduced a general class of variational inequalities known as quasi variational inequality, which has many applications in fluid dynamics, imulse control and genral theory of equilibrium. In a quasi variational inequality formulation, the convex set also depends on the solution. Related to the variational inequality problem, there is also a complementarity problem, which has many applications in operations research, economics equilibrium and management sciences. In fact, it is known that if the underlying set in both these problems is a convex cone, then complementarity problem and variational inequality problem are equivalent. This equivalent has been used quite effectively in suggesting unified and general algorithms for solving complementarity problems, see Ahn $[2]$ and Noor $[3,4,5]$..

Motivated and inpired by the research activities in these fields, we introduce a new class of complementarity problems, which is the natural 
generalization of linear and nonlinear complementarity problems studied extensively in recent years. After introducing the problem, we show that its solution can be obtained from an iterative scheme. We also discuss the convergence criteria for the approximate solution. Several special cases, which can be obtained from our main results, are disucssed. our results are an extension and improvements of the previously known results.

2. PRELIMINARIES AND BASIC RESULTS.

Let $\mathrm{H}$ be a real Hilbert space with its dual H', whose inner product and norm are denoted by (.,.) and $\|$.$\| respectively. We identify the$

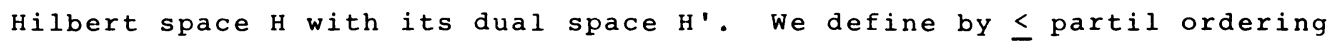
on $\mathrm{H}$, which is consistant with the topological vector structure of $\mathrm{H}$ that is the cone of all non-negative elements $\{v \varepsilon H: v \geq 0\}$ is a closed convex cone. Let $K$ be a closed convex set in $H$. If $T: K \rightarrow H$ is a continuous mapping, then we consider the problem of finding uek such that

$$
(T u, v-u) \geq 0, \quad \text { for all } v \varepsilon K \text {. }
$$

Inequality of type (1.1) is known as variational inequality. If the convex set $K$ also depends upon the solution, then variational inequality (1.1) is called quasi variational inequality. To be more specific, the quasi variational inequality problem is to find uek(u) such that

$$
(T u, v-u) \geq 0, \quad \text { for all } v \in K(u) \text {. }
$$

In many important applications, $K(u)$ has the following form

$$
K(u)=m(u)+K \text {, }
$$

where $m$ is a point-to-point mapping. In this case $K^{\star}(u)=(m(u)+K)^{*}=$ $m(u) \cap k^{*}$. For applications of quasi variational inequalities, see $[6,7,8,9]$.

Related to quasi variational inequalities, we now introduce a new class of complementarity problems, which will be called quasi complementarity problem of finding u such that

$$
u-m(u) \geq 0, \quad T u \geq 0, \quad(u-m(u), T u)=0
$$

If $T$ is a nonlinear transformation, then problem (1.4) is called the nonlinear quasi complementarity problem (NQCP). If $T$ is an affine transformation of the form $u \rightarrow M u+q$, where $M$ is a nxn matrix, $q$ is a n-vector, then problem (1.4) is equivalent to finding u such that

$$
u-m(u) \geq 0, \quad M u+q \geq 0, \quad(u-m(u), \quad M u+q)=0,
$$

which is called linear quasi complementarity problem. Problem of type (1.5) was introduced and studied by Dolcetta [10], Mosco [7] and Pang $[11,12]$.

In particular, if the mapping $m$ is zero, then problems (1.4) and (1.5) are equivalent to the following complementarity problems; 


$$
\mathrm{u} \geq 0, \quad \mathrm{Tu} \geq 0, \quad(\mathrm{u}, \mathrm{Tu})=0
$$

and

$$
u \geq 0, \quad M u+q \geq 0, \quad(u, M u+q)=0,
$$

which have been extensively studied by cottle [13,14], Karamardian [15], Mangasarian [16] and Ahn [17] in recent years.

Let $\mathrm{K}^{*}=\{\mathrm{u} \varepsilon \mathrm{H},(\mathrm{u}, \mathrm{v}) \geq 0$ for all $\mathrm{v} \varepsilon \mathrm{K}\}$, be the polar cone of the convex cone $k$ in $H$. If the convex cone also depends upon the solution itself, then we introduce the generalized quasi complementarity problem of finding $u \varepsilon K(u)$ such that

$$
T u \varepsilon K^{*}(u) \text { and }(u-m(u), T u)=0,
$$

It is clear that the nonlinear quasi complementarity problem (1.4) is a special case of the generalized nonlinear quasi complementarity problme (1.8). Furthermore, if the point-to-point mapping $m$ is zero,then problem (1.8) is equivalent to finding uek such that

$$
\mathrm{Tu} \varepsilon \mathrm{K}^{*} \text { and }(\mathrm{u}, \mathrm{Tu})=0 \text {, }
$$

which is known as the generalized nonlinear complementarity problem considered and studied by Karamardian [15], cottle [14], Noor [3] and many others.

3. MAIN RESULTS.

We need the following results, the first is a generalization of a result of Karamardian [15] and Pang [11].

LEMMA 3.1. If $K$ is the positive cone in $H$, then uEK(u), defined by (1.3), is a solution of (1.8) if and only if u satisfies the quasi variational inequality (1.2).

PROOF. The proof is similar to that of lemma 3.1 in $[18,11]$. THEOREM 3.1 [19,20]. For $K(u)$ given by $(1.3)$, uEK(u) is a solution of quasi variational inequality (1.2) if and only if u satisfies the following relation

$$
u=m(u)+P_{K}[u-\rho(T u)-m(u)]
$$

for some $\rho>0$, where $m$ is an arbitrary point-to-point mapping and $P_{K}$ is the projection of $H$ into $K$, the positive cone in $H$. For the description of $\mathrm{P}_{\mathrm{K}}$, see $[8]$.

From lemma 3.1 and theorem 3.1 , we conclude that the solution to problem (1.8) may be obtained by computing the fixed point of the function defined by (3.1). In fact, theorem 3.1 enables us to find the approximate solution $u_{n}$ by the following iterative scheme:

$$
u_{n+1}=m\left(u_{n}\right)+P_{K}\left[u_{n}-\rho T\left(u_{n}\right)-m\left(u_{n}\right)\right], \quad n=0,1,2, \ldots \text { (3.2) }
$$

We also need the following concepts.

DEFINITION 3.1. A mapping $T: H \rightarrow H$ is said to be

i. Strongly Monotone, if there exists a constant $\alpha>0$ such that $\left\langle\mathrm{Tu}-\mathrm{TV}, \mathrm{u}-\mathrm{v}>\geq \alpha\|\mathrm{u}-\mathrm{v}\|^{2}\right.$, for all $\mathrm{u}, \mathrm{v} \varepsilon \mathrm{H}$. 
ii. Lipschitz Continuous, if there exists a constant $\beta>0$ such that

$$
\|\mathrm{Tu}-\mathrm{Tv}\|<\beta\|\mathrm{u}-\mathrm{v}\| \text {, for all } \mathrm{u}, \mathrm{v} \varepsilon \mathrm{H} \text {. }
$$

In particular, it follows that $\alpha \leq \beta$.

In the next theorem, we study the conditions under which the approximate solution obtained from (3.2) converges to the exact solution u of quasi complementarity problem (QCP, 1.8).

THEOREM 3.2. Let the mapping $T$ be strongly monotone and Lipschitz continuous. If the arbitrary mapping $m$ is Lipschitz continuous, $u_{n}$ and $u$ are solutions satisfying (3.1) and (1.8), then $u_{n}$ converges to u strongly in $\mathrm{H}$, for

$$
\left|\rho-\frac{\alpha}{\beta^{2}}\right|<\frac{\sqrt{\alpha^{2}-4 \beta^{2}\left(\gamma-\gamma^{2}\right)}}{\beta^{2}}, \alpha \geq 2 \beta \sqrt{\gamma-\gamma^{2}} \text { and } \gamma<1 / 2 \text {, }
$$

where $\gamma$ is the Lipschitz constant of $\mathrm{m}$.

PROOF. The method of proof is similar to the one given in [5]. From lemma 3.1 and theorem 3.1 , we observe that the solution u of (1.8) can be characterized by the relation (3.1). Hence from (3.1) and (3.2), we have

$$
\begin{aligned}
\left\|u_{n+1}-u\right\| & \leq\left\|m\left(u_{n}\right)-m(u)+P_{K}\left[u_{n}-\rho\left(T u_{n}\right)-m\left(u_{n}\right)\right]-P_{K}[u-\rho(T u)-m(u)]\right\| \\
& \leq\left\|m\left(u_{n}\right)-m(u)\right\|+\left\|u_{n}-u-\rho\left(T u_{n}-T u\right)-m\left(u_{n}\right)+m(u)\right\|, \\
& \text { since } P_{K} \text { is non-expansive, see }[21,6] . \\
& \leq 2 \gamma\left\|u_{n}-u\right\|+\left\|u_{n}-u-\rho\left(T u_{n}-T u\right)\right\|
\end{aligned}
$$

Now by the strongly monotonicity and Lipschitz continuity of $T$, we obtain

$$
\left\|u_{n}-u-\rho\left(T u_{n}-T u\right)\right\|^{2} \leq\left(1-2 \rho \alpha+\beta^{2} \rho^{2}\right)\left\|u_{n}-u\right\|^{2} \text {. }
$$

Thus using the above inequality, we have

$$
\begin{aligned}
\left\|u_{n+1}-u\right\| & \leq\left[\sqrt{\left(1-2 \alpha \rho+\beta^{2} \rho^{2}\right)}+2 \gamma\right]\left\|u_{n}-u\right\| \\
& \leq \theta\left\|u_{n}-u\right\|,
\end{aligned}
$$

where

$$
\begin{aligned}
& \theta=\left[\sqrt{\left(1-2 \rho \alpha+\beta^{2} \rho^{2}\right)}+2 \gamma\right]<1 \text { for }\left|\rho-\frac{\alpha}{\beta^{2}}\right|<\frac{\sqrt{\alpha^{2}-4 \beta^{2}\left(\gamma-\gamma^{2}\right)}}{\beta^{2}} \text {, } \\
& \gamma<\frac{1}{2} \text { and } \alpha \geq 2 \beta \sqrt{\gamma-\gamma^{2}}
\end{aligned}
$$

Since $\theta<1$, so the fixed point problem (3.1) has a unique solution u and consequently the picard iterate $u_{n+1}$ converges to $u$; see [22], which is the required result.

It is clear that the use of algorithms as constructive methods for proving the existence of solution brings theory and computation closer together. Theorem 3.2 enables us to find the approximate solution of ( 1.8 ) by the following iterative scheme:

$$
\begin{aligned}
& \text { i. Given } u_{0} \varepsilon_{H} \text {. } \\
& \text { ii. } u_{n+1}=m\left(u_{n}\right)+P_{K}\left[u_{n}-\rho\left(T u_{n}\right)-m\left(u_{n}\right)\right], \quad n=0,1,2,3, \ldots
\end{aligned}
$$


where $\mathrm{P}_{\mathrm{K}}$ is the projection of $\mathrm{H}$ into $\mathrm{K}, \mathrm{m}$ is a point-to-point mapping and $\rho>0$ is required to satisfy the following conditions:

$$
\left|\rho-\frac{\alpha}{\beta^{2}}\right|<\frac{\sqrt{\alpha^{2}-4 \beta^{2}\left(\gamma-\gamma^{2}\right)}}{\beta^{2}}, \gamma<\frac{1}{2} \text { and } \alpha \geq 2 \beta \sqrt{\gamma-\gamma^{2}} \text {. }
$$

REMARK 3.1. If the point-to-point mapping is zero, then it implies that the Lipschitz constant $\gamma$ is zero. Thus our algorithm is exactly the same as considered by Fang [23] and Gana [24]. Furthermore, theorem 3.1 reduces to a result of Noor and Noor $[21,25]$ for the corresponding variational inequalities. When $T$ is of the form $T u=M u+q$, one can easily prove that $T$ is strongly monotone if and only if $M$ is positive definite, see [15] for proof. Obviously $T$ is also Lipschitz continuous. For the linear quasi complementarity problem, see [18] where one can find a general algorithm for finding the approximate solution along with the necessary convergence criteria.

ACKNOWLEDGEMENT. I wish to express my gratitude to Prof. Dr. W. Oettli for his valuable suggestions and comments.

\section{REFERENCES}

1. BENSOUSSAN, A. and LIONS, J. Applications des inequations variationelles en controle stochastique, Dunod, Paris, 1978.

2. AHN, B.H. Iterative methods for linear complementarity problems with upper bounds on primary variables, Math. Programming, 26(1983), 295-315.

3. NOOR, M. ASLAM. Generalized nonlinear complementarity problem, J.Nat. Sci. \& Math., 26(1986), 9-20.

4. NOOR M. ASLAM. On the nonlinear complementarity problem, J.Math. Anal. Appl., to appear.

5. NOOR, M. ASLAM. Generalized quasi complementarity problems, $\underline{\text { J.Math. }}$ Anal. Appl. (1986), to appear.

6. BAIOCCHI, C. and CAPELO, A. Variational and quasi variational inequalities, John Wiley and Sons, New York, 1984 .

7. MosCo, U. Implicit variational problems and quasi variational inequalities, Lect. Notes Math., Springer-Verlag, Berlin, 1976.

8. ODEN, J. and KIKUCHI, N. Theory of variational inequalities with applications to problems of flow through porous media, Int. J. Engng. Science, 18(1986), 1173-1284.

9. CRANK, J. Free and moving boundary problems, Calendron press, London, 1984 .

10. DOLCETTA, I. Systemi di complementarita a desequagliance variazionale, Ph.D. Thesis, University of Rome, Italy, 1972.

11. PANG, J.S. On the convergence of a basic iterative method for the implicit complementarity problem, J. Opt. Theor. Appl. $37(1982)$, 149-162.

12. PANG J.S. The implicit complementarity problem in Nonlinear Programming 4 , (ed.) by Mangasarian, Meyer and Robinson, Academic Press, London, (1981), 487-518.

13. COTTLE, R. Complementarity and variational problems, Symposia Math. 19(1976), 177-208. 
14. COTTLE, R. Note on a fundamental theorem in quadratic programming, SIAM J. Appl. Math., $12(1964), 663-665$.

15. KARAMARDIAN, S. Generalized complementarity problem, J. Opt. Theor. Appli. 8(1971), 161-168.

16. MANGASARIAN, O.L. Solution of symmetric linear complementarity problem by iterative methods, J. Opt. Theor. Appl. 22(1976),465485 .

17. AHN B.H. Solution of non symmetric linear complementarity problems by iterative methods, J.Opt. Thoer. Appl. 33(1981), 175-185.

18. NOOR, M. ASLAM. Convergence analysis of the iterative methods for quasi complementarity problems, to appear.

19. NOOR, M. ASLAM. An iterative scheme for a class of quasi variational inequalities, J.Math. Anal. Appl. 110(1985), 463-468.

20. CHAN, P. and PANG, J.S. The generalized quasi variational inequality problem, Math. Op. Res.?(1982), 221-222.

21. NOOR, M. ASLAM and NOOR, K. INAYAT. Iterative methods for variational inequalities and nonlinear programming, Methods Op. Res. 31 (1979), 455-463.

22. ORTEGA, J.M. and RHEINBOLD, W. Iterative solutions of nonlinear equations in several variables, Academic Press, London, 1970.

23. FANG, S.C. An iterative method for generalized complementarity problems, IEEE Trans. Ant. Control, 25(1980), 1225-1227.

24. GANA, A. Studies in complementarity problems, Ph.D. thesis, University of Michigan, U.S.A., 1982 .

25. NOOR, K. INAYAT and NOOR, M. ASLAM. Iterative methods for a class of variational inequalities in Numerical Analysis of singular Peturbation Problems, (ed.) by Hemker and Milier, Academic Press, London, (1979), 441-448. 


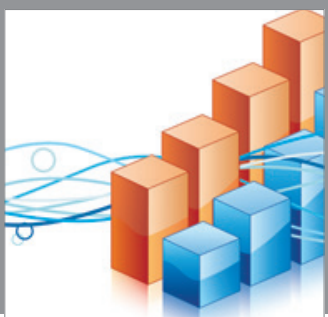

Advances in

Operations Research

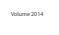

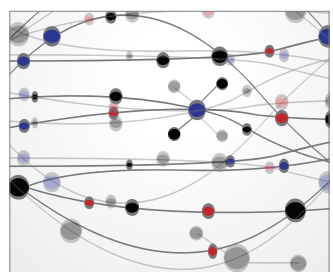

\section{The Scientific} World Journal
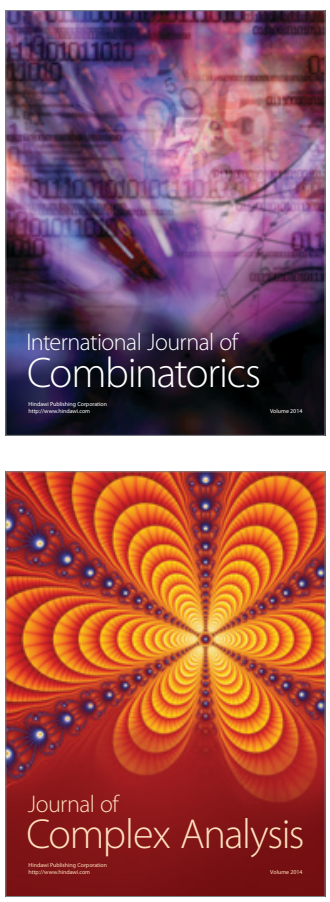

International Journal of

Mathematics and

Mathematical

Sciences
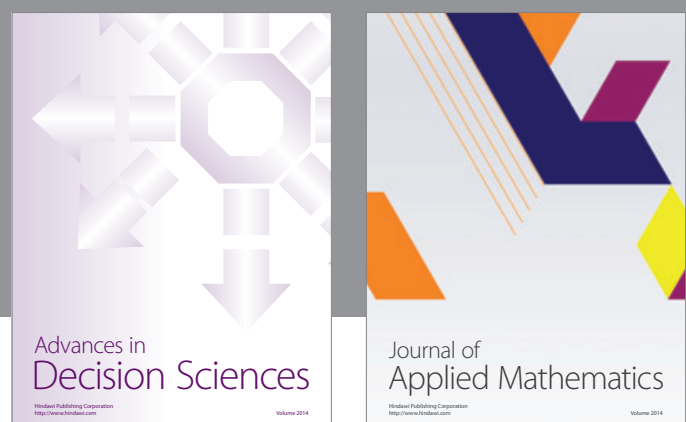

Journal of

Applied Mathematics
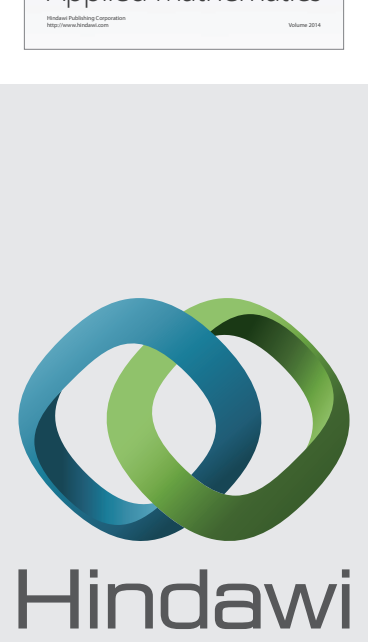

Submit your manuscripts at http://www.hindawi.com
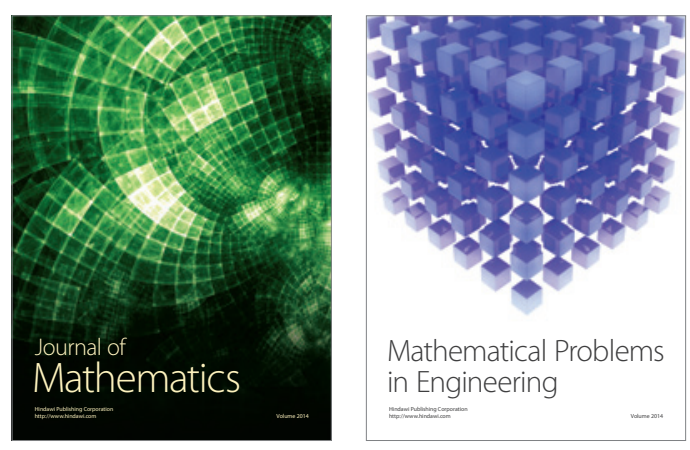

Mathematical Problems in Engineering
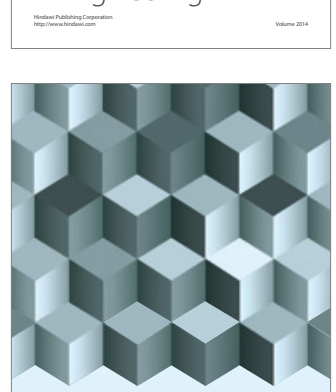

Journal of

Function Spaces
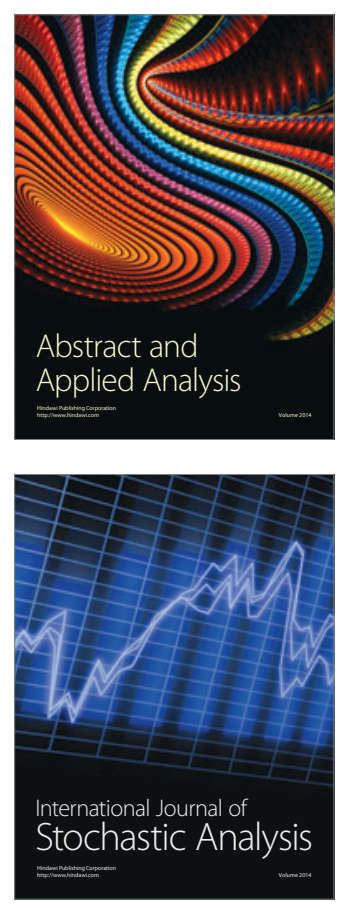

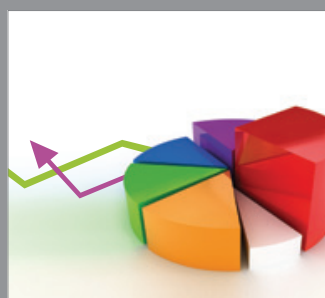

ournal of

Probability and Statistics

Promensencen
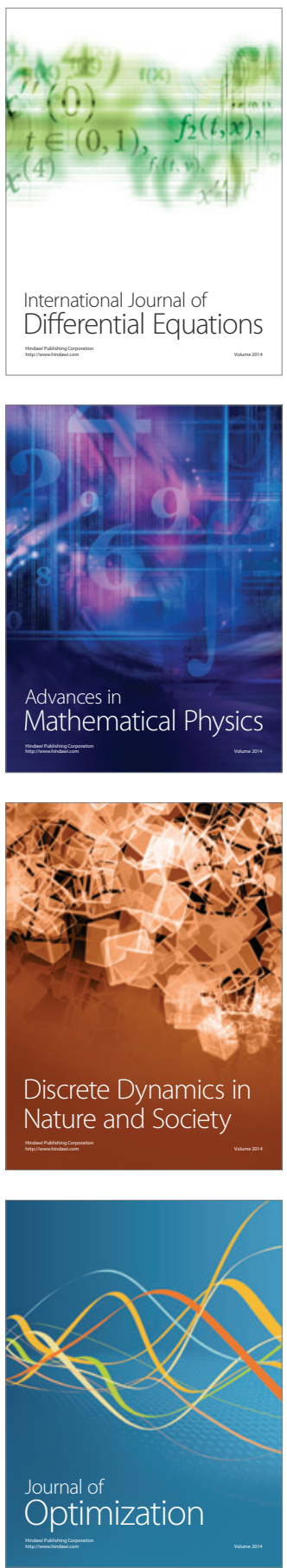